CLINICAL STUDY

\title{
Are short women at risk for gestational diabetes mellitus?
}

\author{
Jarosław Ogonowski and Tomasz Miazgowski ${ }^{1}$ \\ Outpatient Clinic for Diabetic Pregnant Women, 71-455 Szczecin, Poland and ${ }^{1}$ Department of Hypertension and Internal Medicine, Pomeranian Medical \\ University, ul. Unii Lubelskiej 1, 71-252 Szczecin, Poland \\ (Correspondence should be addressed to T Miazgowski; Email: miazgowski@interia.pl)
}

\begin{abstract}
Objective: The aim of the study was to assess the influence of height variations on the risk of gestational diabetes mellitus (GDM).

Research design and methods: We analyzed the medical records of 1830 Caucasian women with GDM and 1011 healthy pregnant women. The following data were collected: age, prior macrosomia, prior GDM, parity, history of type 2 diabetes in first-degree relatives, weight before pregnancy, weight gain during pregnancy, glucose level at the first obstetric visit, results of the glucose challenge test and oral glucose tolerance test (OGTT), HbA1c, and method for treatment of GDM.

Results: Women with GDM were significantly shorter than the healthy controls $(165.7 \pm 5.6$ vs $163.8 \pm 6.6 \mathrm{~cm} ; P<0.001)$. The differences in height were not significant between GDM women who required insulin therapy and those treated with diet alone $(P=0.12)$. All the studied variables, including height, were independently associated with GDM. Even after adjustment for confounding variables, height was still associated with GDM (odds ratio 0.958, 95\% confidence interval: 0.94-0.97; $P<0.00001)$. In women with GDM diagnosed by 75-g OGTT, we found a significant inverse association of height adjusted for age and pregravid weight with 2 -h glucose level $(\beta=-0.12 ; P<0.0001)$. Conclusions: Caucasian women with GDM are shorter than pregnant women without GDM regardless of the diagnostic criteria used or the severity of glucose intolerance. Although height is an independent predictor for GDM, its predictive value for identifying women at risk is relatively low and should not be considered in selective screening for this disease.
\end{abstract}

European Journal of Endocrinology 162 491-497

\section{Introduction}

In 1991, Brown et al. (1) published the first study demonstrating the negative relationship between height and glucose tolerance in adults. Further studies not only confirmed this observation in different ethnic groups with type 2 diabetes (2-5), but also showed that women with gestational diabetes mellitus (GDM) were usually shorter than women without GDM (6-12). This association between short stature and a higher prevalence of both type 2 diabetes and GDM suggests that both forms of glucose intolerance are likely to have a similar pathogenesis characterized by insulin resistance and $\beta$-cell dysfunction $(13,14)$.

The higher prevalence of short stature in women with GDM was noted in both homogeneous $(6,7,10)$ and heterogeneous $(8,9,11,12)$ racial groups, and there was a negative relationship between height and the severity of glucose intolerance. By comparing pregnant women with one abnormal result in the diagnostic oral glucose tolerance test (OGTT) with women diagnosed with either GDM or pregravid type 2 diabetes, Anastasiou et al. (6) showed a linear relationship between decreasing height and increasing frequencies of abnormal glucose tolerance and insulin resistance.
Similarly, other studies demonstrated significant differences in height between healthy pregnant women, women with a false positive OGTT result, and women diagnosed with GDM (7). This direct link between short stature and increased risk for GDM might be clinically relevant, especially in screening strategies based on a selective approach that uses a history of traditional risk factors as a selection for screening. However, implementing these results in clinical practice in Caucasian women seems uncertain mostly due to methodological considerations. Previous studies were performed on either mixed $(8,9,12)$ or non-Caucasian $(6,7,10)$ populations. Therefore, their results may not apply to all populations due to the variations in average height between countries and socioeconomic groups $(8,9)$. The results may also be influenced by variations in the diagnostic methods that have been developed to identify women at risk of GDM.

In Poland, GDM is routinely diagnosed using a glucose challenge test followed by a diagnostic $75-\mathrm{g}$ OGTT according to the World Health Organization (WHO) criteria (15). Many more women with GDM are identified using the WHO criteria, and they have a wider range of blood glucose concentrations than those identified with the criteria recommended by the 
American Diabetes Association (ADA) (16). In general, women with GDM diagnosed using the ADA criteria have higher mean blood glucose concentrations during pregnancy than those diagnosed using the WHO criteria (15-17).

Given this diagnostic variation, we decided to test the following hypotheses: i) height might be a risk factor for abnormal glucose tolerance during pregnancy in a homogeneous group of Polish Caucasian women, and ii) height differences might predict the severity of abnormal glucose tolerance in women with GDM. Based on these assumptions, we also tested for the possibility to include height to the list of traditional risk factors used in selective screening for GDM.

\section{Material and methods}

In this retrospective study, we assessed the influence of height on the risk of GDM by analyzing the medical records of 1830 women with abnormal glucose challenge test who were referred to the Outpatient Clinic for Diabetic Pregnant Women in Szczecin (northwest Poland) from 1999 to 2005 (inclusive).

In Poland, as a routine procedure, GDM is diagnosed by a two-step approach using a glucose challenge test followed by a 75-g OGTT. The challenge test entails oral administration of $50 \mathrm{~g}$ of glucose at 24 to 28 weeks gestation, regardless of the length of time since the last meal, with a measurement of plasma glucose $1 \mathrm{~h}$ later. Women with 1 -h glucose level $>180 \mathrm{mg} / \mathrm{dl}$ $(10.0 \mathrm{mmol} / \mathrm{l})$ in the challenge test were classified as having GDM. Women with plasma glucose concentrations between $140 \mathrm{mg} / \mathrm{dl} \quad(7.8 \mathrm{mmol} / \mathrm{l})$ and $180 \mathrm{mg} / \mathrm{dl}$ ( $10.0 \mathrm{mmol} / \mathrm{l})$ were referred for a diagnostic $75-\mathrm{g}$ OGTT. Based on the results of the diagnostic OGTT, GDM was diagnosed if either the fasting glucose level was $\geq 110 \mathrm{mg} / \mathrm{dl}(6.1 \mathrm{mmol} / \mathrm{l})$ or the 2 -h glucose concentration was $\geq 140 \mathrm{mg} / \mathrm{dl}(7.8 \mathrm{mmol} / \mathrm{l})$, consistent with the WHO criteria (15). Additionally, we also used the diagnostic criteria for GDM recommended by the ADA (16), where at least two of the three following values were above the reference range: fasting glucose level $\geq 95 \mathrm{mg} / \mathrm{dl}$ ( $5.3 \mathrm{mmol} / \mathrm{l}), 1$-h glucose level $\geq 180 \mathrm{mg} / \mathrm{dl}$ $(10.0 \mathrm{mmol} / \mathrm{l})$, and $2-\mathrm{h}$ glucose level $\geq 155 \mathrm{mg} / \mathrm{dl}$ $(8.6 \mathrm{mmol} / \mathrm{l})$. Women with pregravid diabetes or multiple pregnancies were excluded from further analyses.

The control group consisted of 1011 healthy women with a single pregnancy and a normal challenge test. Women were considered healthy if they did not have a present or past history of medical conditions requiring treatment, declared no history of alcohol or narcotic abuse, and showed no abnormalities upon physical examination. The protocol was approved by the institutional review board.

The following data were collected for all women: age, prior macrosomia, prior GDM, parity, history of type 2 diabetes in first-degree relatives, weight before pregnancy, weight gain during pregnancy, glucose level at the first obstetric visit, results of the glucose challenge test and OGTT, HbAlc, and method for treatment of GDM.

Body mass index (BMI) was calculated from the most recent weight $(\mathrm{kg})$ before conception, and height $(\mathrm{m})$ was measured with a single fixed stadiometer.

All women with GDM were educated and motivated by personnel trained in patient education. The patient education skills of the staff were reviewed, evaluated, and reinforced on a regular basis. Women were trained in achieving effective self-monitoring of their blood glucose levels by four daily measurements (fasting and $1 \mathrm{~h}$ after main meals) and self-adjustment of insulin dose in case of inadequate glycemic control. The treatment regimen included diet, insulin neural protamine hagedorn $(\mathrm{NPH})$ given at bedtime, and/or short-acting human insulin before meals. The therapeutic goals were fasting blood glucose of $60-90 \mathrm{mg} / \mathrm{dl}$ (3.3-5.0 mmol/l) and blood glucose below $130 \mathrm{mg} / \mathrm{dl}$ $(7.3 \mathrm{mmol} / \mathrm{l})$ after meals. These goals were evaluated at each weekly visit.

\section{Statistical analysis}

The distribution of continuous variables was tested for normality by the Shapiro-Wilk test. Comparisons between groups for study variables were done using one-way ANOVA followed by post tests. Unpaired $t$-tests and $\chi^{2}$ test were used to compare baseline characteristics between women with and without GDM. Univariate and multivariate logistic regression analyses were used to explore the associations between risk factors (age, height, BMI, family history of type 2 diabetes, prior macrosomia, and prior GDM) and a diagnosis of GDM. The results of the analyses are expressed as odds ratios (ORs) and 95\% confidence intervals (CIs). For determination of a cut-off value for height, the coordinate of a receiver operating characteristic (ROC) curve was used. Linear correlation and regression or non-parametric regression analyses were used to test for relationship between height and serum glucose level during OGTT.

\section{Results}

Of all 1830 women with abnormal result of the challenge test, GDM was diagnosed in 293 women (16\%) solely by a positive challenge test. In the remaining group of women $(84 \%)$, a 75 -g OGTT was performed. The WHO criteria diagnosed GDM in 1121 (61\%) women, whereas $416(23 \%)$ pregnant women had a normal diagnostic OGTT (false positive group). Among women with GDM (according to the WHO diagnostic criteria), 429 women (23\%) also fulfilled the ADA diagnostic criteria for GDM. Overall, of all women with abnormal results of the challenge test, we identified 1414 women $(77 \%)$ with GDM. The screening algorithm is depicted in Fig. 1. 


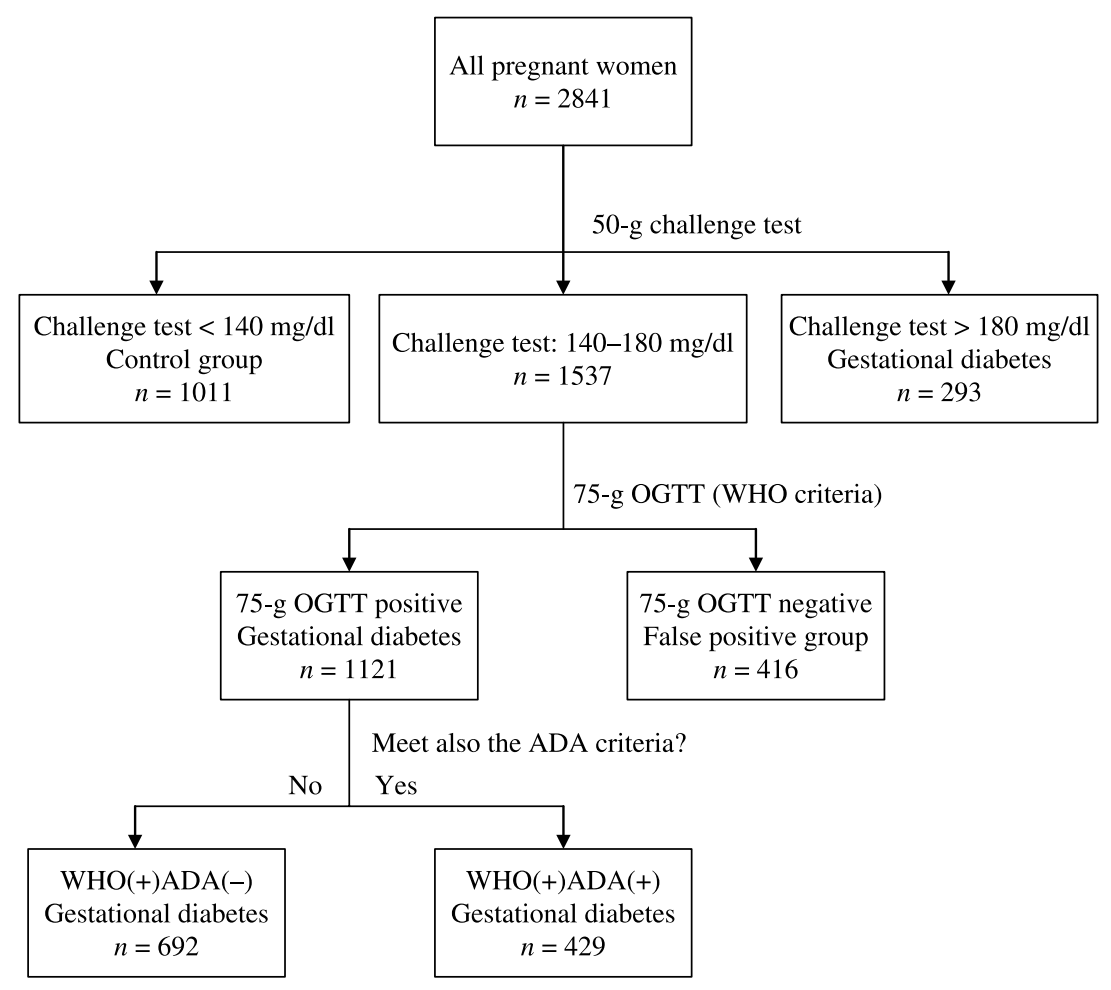

Figure 1 Screening algorithm for gestational diabetes mellitus.
Baseline clinical and anthropometric characteristics are given in Table 1. In comparison with the controls, women with GDM were significantly older and shorter, and had higher pregravid weight, BMI, and glucose level both at the first obstetric visit and during the challenge test. While comparable differences were found between the control group and the false positive group, both of these groups had a similar average height. Women with GDM frequently had a personal history of macrosomia and prior GDM and a family history of type 2 diabetes.

Through additional application of the ADA criteria, two groups of women with GDM were identified: those who fulfilled both diagnostic criteria $(\mathrm{WHO}(+) \mathrm{ADA}(+))$ and women who fulfilled the WHO criteria but not the ADA criteria $(\mathrm{WHO}(+) \mathrm{ADA}(-))$. Thus, we analyzed groups with an increasing severity of GDM: the controls $<$ false positive $<\mathrm{WHO}(+) \mathrm{ADA}(-)<\mathrm{WHO}(+) \mathrm{ADA}(+)$ $<$ challenge test positive. Table 2 summarizes the parameters of glucose tolerance in these groups of women. As expected, most severe abnormalities of glucose metabolism were found in women with a positive challenge test and in the $\mathrm{WHO}(+) \mathrm{ADA}(+)$ group. In both groups, a similar percentage of women required insulin therapy, whereas the requirement of insulin therapy was rare for $\mathrm{WHO}(+) \mathrm{ADA}(-)$ women. In comparison with the controls, pregnant women with a false positive test had higher plasma glucose both when fasting $(P<0.001)$ and during the challenge test $(P<0.001)$. Women diagnosed by the positive challenge test only represented the most pronounced abnormal glucose tolerance. These women had significantly higher HbA1c $(P<0.001)$, challenge test results $(P<0.001)$, and fasting plasma glucose levels $(P<0.001)$ compared to the $\mathrm{WHO}(+) \mathrm{ADA}(+)$ and $\mathrm{WHO}(+) \mathrm{ADA}(-)$ groups.

As shown in Fig. 2, pregravid BMI and height are associated with the severity of glucose intolerance during pregnancy. Regardless of the criteria used for diagnosis, women with GDM were significantly shorter

Table 1 Baseline characteristics of the women with gestational diabetes mellitus, those with a false positive challenge test, and the controls.

\begin{tabular}{|c|c|c|c|}
\hline & $\begin{array}{l}\text { Controls } \\
(n=1011)\end{array}$ & $\begin{array}{l}\text { Women with a } \\
\text { false positive } \\
\text { challenge test } \\
\quad(n=416)\end{array}$ & $\begin{array}{c}\text { Women with } \\
\text { gestational } \\
\text { diabetes } \\
(n=1414)\end{array}$ \\
\hline Age (years) & $27.5 \pm 4.7$ & $28.3 \pm 5.3^{\star}$ & $30.2 \pm 5.6^{*, \dagger}$ \\
\hline $\begin{array}{l}\text { Pregravid body } \\
\text { mass index }\left(\mathrm{kg} / \mathrm{m}^{2}\right)\end{array}$ & $21.9 \pm 3.6$ & $23.5 \pm 4.5^{\star}$ & $24.23 \pm 5.2^{*, \dagger}$ \\
\hline Height $(\mathrm{cm})$ & $165.7 \pm 5.6$ & $165.4 \pm 5.8$ & $163.8 \pm 6.6^{*, \dagger}$ \\
\hline $\begin{array}{l}\text { Weight before } \\
\text { pregnancy }(\mathrm{kg})\end{array}$ & $60.3 \pm 10.6$ & $64.5 \pm 13.6^{\star}$ & $64.8 \pm 14.7^{*}$ \\
\hline $\begin{array}{l}\text { Prior gestational } \\
\text { diabetes }(n)\end{array}$ & $3(0.3 \%)$ & $11(2.7 \%)$ & $75(5.3 \%)^{\ddagger}$ \\
\hline Multiparity $(n)$ & $425(42.0 \%)$ & 219 (52.8\%) & $806(57.0)^{\ddagger}$ \\
\hline $\begin{array}{l}\text { Prior } \\
\quad \text { macrosomia }(n)\end{array}$ & $32(3.2 \%)$ & $28(6.7 \%)$ & $151(10.7)^{\ddagger}$ \\
\hline $\begin{array}{l}\text { Family history of } \\
\text { type } 2 \text { diabetes }(n)\end{array}$ & $52(5.1 \%)$ & $112(27.0 \%)$ & $433(30.6)^{\ddagger}$ \\
\hline
\end{tabular}

Data are means \pm s.D. ${ }^{*} P<0.001$ compared to the controls; ${ }^{\dagger} P<0.001$ compared to the false positive group; ${ }^{\ddagger} P<0.00001$ ( $\chi^{2}$ test). 
Table 2 Glucose tolerance parameters in pregnant women with different degrees of glucose intolerance and the healthy controls.

\begin{tabular}{lccccc}
\hline & $\begin{array}{c}\text { Controls } \\
(n=1011)\end{array}$ & $\begin{array}{c}\text { False positive } \\
(n=416)\end{array}$ & $\begin{array}{c}\text { WHO(+)ADA(-) } \\
(n=692)\end{array}$ & $\begin{array}{c}\text { WHO(+)ADA(+) } \\
(n=429)\end{array}$ & $\begin{array}{c}\text { Challenge test } \\
\text { positive }(n=293)\end{array}$ \\
\hline HbA1c (\%) & & $4.98 \pm 0.44$ & $5.11 \pm 0.47$ & $5.16 \pm 0.54$ & $5.20 \pm 0.61^{*}$ \\
Fasting plasma glucose (mg/dl) & $80.6 \pm 10.1$ & $85.8 \pm 12.7$ & $85.9 \pm 12.9$ & $93.3 \pm 16.6$ & $95.2 \pm 20.7^{\dagger}$ \\
Challenge test 60 min (mg/dl) & $105.5 \pm 19.6$ & $159.2 \pm 14.1$ & $165.9 \pm 19.3$ & $171.2 \pm 21.5$ & $202.5 \pm 18.9^{\dagger}$ \\
OGTT 0 min (mg/dl) & & $78.6 \pm 9.9$ & $84.2 \pm 12.5$ & $99.6 \pm 15.5^{\star}$ & \\
OGTT 60 min (mg/dl) & $140.9 \pm 28.8$ & $161.2 \pm 22.0$ & $199.7 \pm 27.2^{*}$ & \\
OGTT 120 min (mg/dl) & $109.8 \pm 19.4$ & $157.1 \pm 20.4$ & $170.8 \pm 27.6^{*}$ & $120(40.8)^{\ddagger}$ \\
Insulin treated (n) & & $136(19.7 \%)$ & $160(37.3 \%)$ & $1 \%$ \\
\hline
\end{tabular}

Data are means \pm s.D. OGTT, oral glucose tolerance test; WHO, positive $(+)$ or negative $(-)$ to the World Health Organization diagnostic criteria; ADA, positive $(+)$ or negative $(-)$ to the American Diabetes Association diagnostic criteria for gestational diabetes. ${ }^{\star} P<0.001$ versus the false positive group; ${ }^{\dagger} P<0.001$ versus the control group; ${ }^{\ddagger} P<0.001$ versus the $\mathrm{WHO}(+) \mathrm{ADA}(-)$ group.

than the healthy controls. However, despite differences in the severity of glucose intolerance, height did not differ significantly between the GDM groups. The differences in height were not significant between GDM women who required insulin therapy and those treated with diet alone $(163.3 \pm 7.6$ and $164.1 \pm 6.1 \mathrm{~cm}$ respectively; $P=0.12$ ). However, there was a relationship between pregravid BMI and the severity of glucose intolerance. Furthermore, women treated with insulin had a higher BMI than women treated with diet only $(26.02 \pm 5.7$ vs $23.4 \pm 4.8 ; P<0.001)$.

In the univariate logistic regression analysis, height was a strong predictor for GDM, and an increase in height by $1 \mathrm{~cm}$ was associated with a $4.8 \%$ decreased risk for GDM (OR 0.952, 95\% CI: 0.94-0.96; $P<0.00001)$. Multivariate logistic regression analysis was performed to identify significant and independent factors that increase risk for GDM. The variables included
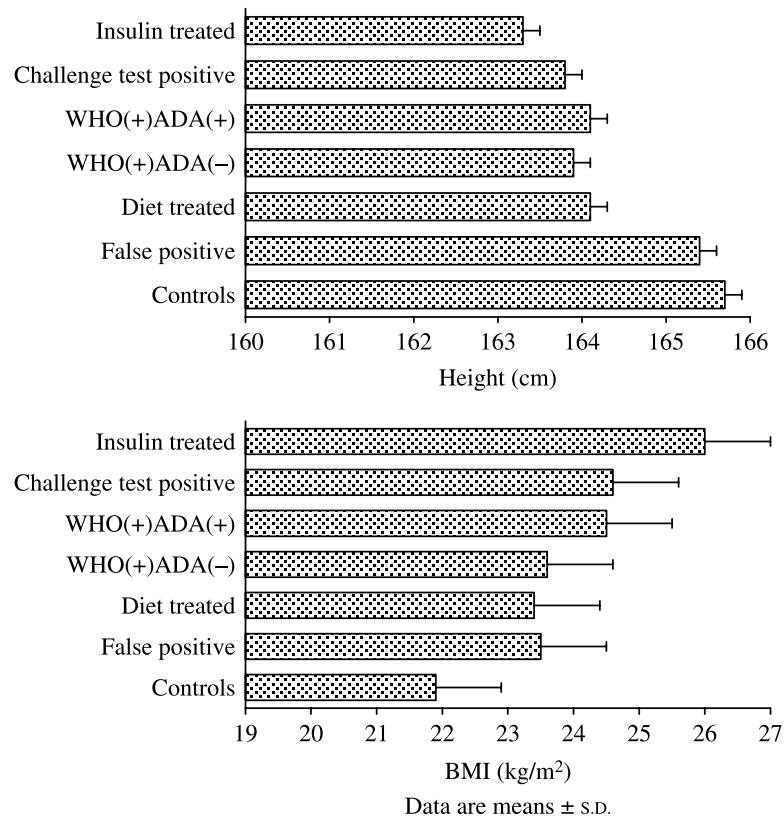

Figure 2 Height and pregravid body mass index in relation to severity of glucose intolerance. in the analysis were age, weight before pregnancy, height, weight gain during pregnancy, prior GDM, prior macrosomia, and family history of type 2 diabetes (Table 3). All the studied variables, including height, were independently associated with GDM. Even after adjustment for confounding variables, height was still associated with GDM (OR 0.958, 95\% CI: 0.94-0.97; $P<0.00001)$. Therefore, we calculated the ROC in order to define a cut-off value for height, below which the risk of GDM substantially increases. The calculated cut-off value was $163 \mathrm{~cm}$. However, the area under the curve was $0.4188 \pm 0.0107$, which did not allow for assumption of this value as predictive for GDM due to a poor discriminatory power. Moreover, cut-off sensitivity and specificity were also low (42.7 and $46.6 \%$ respectively). In all women who underwent a $75-\mathrm{g}$ OGTT, there was a weak but significant inverse correlation between height and 2-h glucose level $(R=-0.13 ; P<0.0001)$, but not with the fasting glucose level $(R=-0.04 ; P=0.12)$. Similarly, a significant inverse association of height adjusted for age and pregravid weight with 2 -h glucose level was found $(\beta=-0.12 ; P<0.0001)$.

\section{Discussion}

This retrospective study in a Polish Caucasian population demonstrates that height is associated with a risk of GDM, and that women with GDM are shorter than healthy pregnant women. Our results also indicate that the height of women with GDM was similar despite different severities of glucose tolerance and different treatment regimens. These findings are partially consistent with earlier studies demonstrating significant differences in the height of women with and without GDM (6-12). Although this phenomenon has not yet been fully explained, there is possibly a direct link between the components of height and abnormal glucose tolerance. Asao et al. (4) have demonstrated that in the general US population, short legs and a low leg length to height ratio are commonly associated with obesity, insulin resistance, and type 2 diabetes. The British Women's Heart and Health Study (5) also 
Table 3 Multiple logistic regression analysis with gestational diabetes mellitus as a dependent variable.

\begin{tabular}{lccc}
\hline & $\begin{array}{l}\text { Odds } \\
\text { ratio }\end{array}$ & $\mathbf{9 5 \%}$ Cl & $\boldsymbol{P}$ \\
\hline Age & 1.087 & $1.07-1.11$ & 0.000001 \\
Weight before pregnancy & 1.071 & $1.05-1.09$ & 0.000001 \\
Height & 0.957 & $0.94-0.98$ & 0.000001 \\
Weight gain during pregnancy & 1.045 & $1.03-1.07$ & 0.000003 \\
Previous gestational diabetes & 5.101 & $2.67-9.76$ & 0.000001 \\
Previous macrosomia & 1.515 & $1.03-2.23$ & 0.00346 \\
Family history of diabetes & 2.866 & $2.28-3.59$ & 0.000001 \\
\hline
\end{tabular}

demonstrated that decreased leg length or leg to trunk length ratio was associated with type 2 diabetes and insulin resistance in older women. Short legs and a low leg to trunk ratio in adults may reflect abnormal growth during childhood, and adult leg length can be used as an indicator of infant nutrition $(18,19)$. Individuals who were breastfed in infancy and were well fed in the first 4 years of childhood were shown to have longer legs in adulthood $(19,20)$ and a lower risk for type 2 diabetes $(20,21)$. Earlier attempts to understand the relationship between short stature and glucose intolerance have considered at least three essential factors: genetic predisposition, influence of the intra-uterine environment on fetal metabolic programming (which in turn affects both height and diabetes risk $(21,22))$, and poor socioeconomic circumstances during childhood $(6,7,9)$. On the other hand, recent studies have suggested an interesting hypothesis that the variations in height might influence the OGTT results. It was demonstrated that among subjects who underwent a 75-g OGTT, women had higher 2-h plasma glucose levels than men. However, this gender difference disappeared after adjusting for height (23-25). This relationship between height and 2-h glucose levels might be explained by the different metabolism of a standard dose of glucose during OGTT in shorter and taller subjects. Subjects with a short stature may have impaired metabolism of $75 \mathrm{~g}$ glucose in comparison with tall individuals because of a lower mass of metabolically active tissues, predominantly fat-free mass (24). Therefore, the incidence of diabetes mellitus and impaired glucose tolerance in individuals with a short stature may be overestimated. This is in line with our findings because in our cohort short stature was significantly associated with a higher 2-h glucose value. To elucidate this phenomenon, further studies on the metabolic and anthropometric determinants of glucose metabolism are necessary.

In our study, we used the different diagnostic criteria and different treatment regimens to stratify women with GDM into subgroups that reflected the severity of glucose intolerance. Interestingly, while women with GDM were significantly shorter than the controls or women with a false positive challenge test, increased GDM severity was not associated with a decrease in height. Similarly, women treated with diet alone had an average height that was comparable to that of the women who required additional treatment with insulin. This is the first study to evaluate the association between short stature and severity of GDM in Caucasian women. In a sample of Greek pregnant women with various forms of abnormal glucose tolerance (pregravid type 1 and type 2 diabetes, GDM, or one abnormal glucose value in a 100-g OGTT), Anastasiou et al. (6) found that there was a gradual decrease in mean height with worsening glucose intolerance. A similar trend, but not associated with different diagnostic criteria for GDM, was observed in Korean (7) and Brazilian (9) women with GDM. Interestingly, our study found that while the height of women with any degree of glucose intolerance did differ significantly, women with more severe glucose intolerance were significantly heavier before pregnancy, leading to a higher pregravid BMI. Body mass-related insulin resistance was associated with both more severe hyperglycemia and an insulin therapy requirement. We also found that women with a false positive challenge test had an average height that was similar to that of the healthy controls, which is inconsistent with previous studies reporting a decreased average height in women with a false positive screening test (1-h plasma glucose $\geq 7.2 \mathrm{mmol} / \mathrm{l}$ ) (7) or one abnormal value in a 100-g OGTT (6). These discrepancies seem to reflect anthropometric variations among ethnic groups, as Polish Caucasian women are taller than Korean or Mediterranean women. Moreover, application of different diagnostic criteria for GDM would also affect the results, as using the National Diabetes Data Group GDM criteria may lead to the underdiagnosis of the condition by excluding women with relatively mild glucose intolerance, especially in groups with a false positive screening test. The problem of different diagnostic criteria for GDM also applies to our study. If we used only the ADA criteria, women classified as $\mathrm{WHO}(+) \mathrm{ADA}(-)$ would have been considered false positives instead of being treated. However, these women had different anthropometric characteristics and glucose metabolism parameters than the false positive group under the WHO criteria, and as much as $20 \%$ of the $\mathrm{WHO}(+) \mathrm{ADA}(-)$ women required insulin therapy.

Univariate and multivariate logistic regression analyses showed that height was an independent predictor of abnormal glucose tolerance during pregnancy, consistent with previous research $(7,10)$. However, the clinical relevance of this is unclear. The calculated cut-off value for height in our sample $(163 \mathrm{~cm})$ had low specificity and sensitivity, meaning its discriminating value seems to be relatively weak. BMI better identified subjects at risk for GDM and type 2 diabetes than short stature $(22,26)$. The mean height differs significantly among different ethnic and socioeconomic groups. Therefore, short stature will not likely be considered a risk factor when screening for GDM. 
By using a two-step diagnostic algorithm, we identified a false positive group of women. On the basis of current knowledge, these women might be considered as normal glucose-tolerant subjects. However, in the present study, in comparison with the controls, women with a false positive challenge test were significantly heavier, had higher (although still within the normal range) fasting glucose level, and more frequently had a family history of type 2 diabetes and prior GDM. There is no widely accepted method to manage such cases during pregnancy. While many obstetric providers treat false positive women with more intensive observation or therapy, identifying these patients as 'glucose intolerant' or 'borderline diabetes', others maintain that such patients do not warrant additional therapies (27). However, there is an increasing body of evidence that women with a false positive test are at risk not only for developing diabetes in the future but also for adverse perinatal outcome, including overall perinatal adversity, endometritis, shoulder dystocia, fetal macrosomia, and antenatal death $(27,28)$. Before changes in clinical practice can be recommended, further studies including cost-benefit analysis of providing additional therapy to these pregnant women are needed.

There are some obvious limitations of our retrospective study. First, we had no patient data from childhood, such as birth weight and length, and were thus unable to determine the independent contributors of pre- versus postnatal factors in glucose tolerance in adulthood. Secondly, we did not identify a group of women diagnosed with GDM using only the ADA criteria. Given that as few as $18 \%$ of pregnant women with GDM (as diagnosed by both the ADA and WHO criteria or the WHO criteria only) fulfill the ADA criteria (17), analyzing a group that used only this diagnostic criteria seems relevant.

In conclusion, Caucasian women with GDM are shorter than pregnant women without GDM regardless of the diagnostic criteria used or the severity of glucose intolerance. Although height is an independent predictor for GDM, its predictive value for identifying women at risk is relatively low, and should not be considered in selective screening for this disease.

\section{Declaration of interest}

The authors declare that there is no conflict of interest that could be perceived as prejudicing the impartiality of the research reported.

\section{Funding}

This research did not receive any specific grant from any funding agency in the public, commercial, or not-for-profit sector.

\section{References}

1 Brown DC, Byrne CD, Clark PMS, Cox BD, Day NE, Hales CN, Shackleton JR, Wang TWM \& Williams DRR. Height and glucose tolerance in adult subjects. Diabetologia $199134531-533$.
2 Riste L, Khan F \& Cruickshank K. High prevalence of type 2 diabetes in all ethnic groups, including Europeans, in a British inner city: relative poverty, history, inactivity or 21st century Europe? Diabetes Care 200124 1377-1383.

3 Jarrett RJ \& Fitzgerald AP. Height and glucose tolerance. Diabetologia 199135 191-192.

4 Asao K, Kao WHL, Baptiste-Roberts K, Bandeen-Roche K, Berlinger TP \& Brancati FL. Short stature and the risk of adiposity, insulin resistance, and type 2 diabetes in middle age. The Third National Health and Nutrition Examination Survey (NHANES III), 1988-1984. Diabetes Care 200629 1632-1637.

5 Lawlor DA, Ibrahim S \& Smith GD. The association between components of adult height and type II diabetes and insulin resistance; British Women's Heart and Health Study. Diabetologia 200245 1097-1106.

6 Anastasiou E, Alevizaki M, Grigorakis SJ, Philippou G, Kyprianou M \& Souvatzoglou A. Decreased stature in gestational diabetes mellitus. Diabetologia 199841 997-1001.

7 Jang HC, Min HK, Lee HK, Cho NH \& Metzger BE. Short stature in Korean women: a contribution to the multifactor predisposition to gestational diabetes mellitus. Diabetologia 199841 778-783.

8 Kousta E, Lawrence NJ, Penny A, Millauer BA, Robinson S, Johnson DG \& McCarthy MI. Women with a history of gestational diabetes of European and South Asian origin are shorter than women with normal glucose tolerance in pregnancy. Diabetic Medicine 200017 792-797.

9 Branchtein L, Schmidt MI, Matos MCG, Yamashita T, Pousada JMDC, Duncan BB \& for the Brazilian Gestational Diabetes Study Group. Short stature and gestational diabetes in Brazil. Diabetologia 200043 848-851.

10 Yang X, Hsu-Hage B, Zhang H, Yu L, Dong L, Li J, Shao P \& Zhang C. Gestational diabetes mellitus in women of single gravidity in Tianjin City, China. Diabetes Care 200225 847-851.

11 Moses RG \& Mackay MT. Gestational diabetes. Is there a relationship between leg length and glucose tolerance? Diabetes Care 200427 1033-1035.

12 Rudra CB, Sorensen TK, Leisenring WM, Dashow E \& Williams MA. Weight characteristic and height in relation to risk of gestational diabetes mellitus. American Journal of Epidemiology $2007165302-308$.

13 Buchanan TA \& Xiang AH. Gestational diabetes mellitus. Journal of Clinical Investigation $2005 \mathbf{1 1 5} 485-491$.

14 Khan SE. The relative contributions of insulin resistance and betacell dysfunction to the pathophysiology of type 2 diabetes. Diabetologia 200346 3-19.

15 WHO Consultation. Definition, Diagnosis and Classification of Diabetes Mellitus and its Complications. Report of a WHO Consultation. Art 1: Diagnosis and Classification of Diabetes Mellitus. Geneva, WHO/NCD/NCS/99.2, World Health Organization, 1999.

16 American Diabetes Association. Gestational diabetes mellitus (Position Statement). Diabetes Care 200225 S94-S96.

17 Schmidt MI, Duncan BB, Reichelt AJ, Branchtein L, Matos MC, Costa e Forti A, Spichler ER, Pousada JM, Teixeria MM, Yamashita T \& Brazilian Gestational Diabetes Study Group . Gestational diabetes mellitus diagnosed with a 2-h 75-g oral glucose tolerance test and adverse pregnancy outcomes. Diabetes Care 200124 1151-1155.

18 Gunnell D, Smith GD, McConnachie A, Greenwood R, Upton M \& Frankel S. Separating in-utero and postnatal influence on later disease. Lancet $19993541526-1527$.

19 Wadsworth MEJ, Hardy RJ, Paul AA, Marshall SF \& Cole TJ. Leg and trunk length at 43 years in relation to childhood health, diet and family circumstances; evidence from the 1946 national birth cohort. International Journal of Epidemiology 200231 383-390.

20 Pettitt DJ, Forman DR, Hanson RL, Knowler WC \& Bennett PH. Breastfeeding and incidence of non-insulin-dependent diabetes mellitus in Pima Indians. Lancet 1997350 166-168.

21 Barker DJ, Gluckman PD, Godfrey KM, Harding JE, Owens JA \& Robinson JS. Fetal nutrition and cardiovascular disease in adult life. Lancet $1993341938-941$. 
22 Di Cianni G, Volpe L, Lencioni C, Miccoli R, Cuccuru I, Ghio A, Chatzianagnostou K, Bottone P, Teti G, Del Prato S \& Benzi L. Prevalence and risk factors for gestational diabetes assessed by universal screening. Diabetes Research and Clinical Practice 200362 131-137.

23 Sicree RA, Zimmet PZ, Dunstan DW, Cameron AJ, Welborn TA \& Shaw JE. Differences in height explain gender differences in the response to oral glucose tolerance test - the AusDiab study. Diabetic Medicine 200825 296-302.

24 Rathmann W, Strassburger K, Giani G, Dốring A \& Meisinger C. Differences in height explain gender differences in the response to oral glucose tolerance test. Diabetic Medicine 200825 1374-1375.

25 Janghorbani M \& Amini M. Effects of gender and height on the oral glucose tolerance test: the Isfahan diabetes prevention study. Review of Diabetic Studies 20085 163-170.
26 Schmidt MI, Matos MC, Reichelt AJ, Forti AC, de Lima L \& Duncan BB for Brazilian Gestational Diabetes Study Group. Prevalence of gestational diabetes mellitus - do the new WHO criteria make a difference? Diabetic Medicine 200017 376-380.

27 Stamilio DM, Olsen T, Ratcliffe S, Sehdev HM \& Macones GA. Falsepositive 1-hour glucose challenge test and adverse perinatal outcomes. Obstetrics and Gynecology 2004103 148-156.

28 Cheng YW, McLaughlin GB, Esakoff TF, Block-Kurbisch I \& Caughey AB. Glucose challenge test: screening threshold for gestational diabetes mellitus and associated outcomes. Journal of Maternal-Fetal and Neonatal Medicine 2007 120 903-908.

Received 26 November 2009

Accepted 30 November 2009 